\title{
Antenna Design for Semi-Passive UHF RFID Transponder with Energy Harvester
}

\author{
Piotr JANKOWSKI-MIHUŁOWICZ, Damian KAWALEC, Mariusz WEGGARSKI
}

Dept. of Electronic and Communications Systems, Rzeszów University of Technology, Pola 2, 35-959 Rzeszów, Poland

pjanko@prz.edu.pl,damkaw@prz.edu.pl,wmar@prz.edu.pl

\begin{abstract}
A novel microstrip antenna which is dedicated to UHF semi-passive RFID transponders with an energy harvester is presented in this paper. The antenna structure designed and simulated by using Mentor Graphics HyperLynx 3D EM software is described in details. The modeling and simulation results along with comparison with experimental data are analyzed and concluded. The main goal of the project is the need to eliminate a traditional battery form the transponder structure. The energy harvesting block, which is used instead, converts ambient energy (electromagnetic energy of typical radio communication system) into electrical power for internal circuitry. The additional function (gathering extra energy) of the transponder antenna causes the necessity to create new designs in this scope.
\end{abstract}

\section{Keywords}

Antenna design, semi-passive chip, RFID technique, microstrip antenna, energy harvesting

\section{Introduction}

The radio frequency identification (RFID) technique is often used in automated identification processes even at the present stage of its development [1]. It is implemented in complex hardware and software systems which have numerous applications in industry, commerce, science, medicine and many other fields [2-6].

In terms of hardware, an RFID system consists of a $\mathrm{read} /$ write device (RWD), its antenna and at least one transponder that is intended for marking an object. Communication in this system is provided with one or with multiple transponders simultaneously (single/anti-collision system). The marked objects during identification process may be placed in fixed point (static system) or may dynamically change their location (dynamic system) [7]. The interrogation zone (IZ) is the main parameter of the RFID system - it determines the efficiency of a given automated process [1], [7]. The size of the interrogation zone strongly depends on the above mentioned arrangements of RWD and transponders. It should be emphasized that there is no universal transponder at the commercial market that can be used for identifying any object in any automated process. In addition, the details about constructional parameters of transponders (of their antennas and used chips) are usually not specified in producers' datasheets. It does not allow designers to estimate precisely IZ and the trial and error method has to be used during implementing an RFID system.

The process of transponder designing always starts with elaborating the antenna that should be matched to the chip to be selected. The application requirements, specific characteristics of target objects, frequency bands etc. should be taken into account. In the literature, it is easy to find antenna designs for classic UHF passive transponders [8-12]. However, the progress in electronics enables to crate new solutions in RFID chips (as internal harvester for additional energy, for example). It is the reason why the precise design guidelines have to be elaborated and new antennas have to be designed.

The modern design of semi-passive UHF transponder with an energy harvester is characterized in Sec. 2. The process of antenna synthesis for rare but commercially available type of chips is discussed in Sec. 3. The particular attention was paid to the method of matching antenna and chip impedances (Sec. 3.1) and antenna construction (Sec. 3.2). The model of antenna was carried out in the Mentor Graphics HyperLynx 3D EM (HL3DEM) software tool and the adequate assumptions are described in Sec. 3.3. The practical implementation was made in PCB technology (Sec. 3.4). The work is summarized by the comparison of calculations and measurements (Sec. 3.5).

\section{Semi-Passive UHF Transponder}

Radiative coupled RFID systems in the UHF band depending on the region of the world - typically operate in the range from $860 \mathrm{MHz}$ to $960 \mathrm{MHz}$. Appropriate communication mechanisms are defined by the ISO/IEC 18000-6 protocol (EPC Class 1 Gen 2 [13]).

The most popular passive RFID transponder contains only the chip with the connected antenna (Fig. 1). Access to the internal memory of the chip can be done only by using the radio interface. 


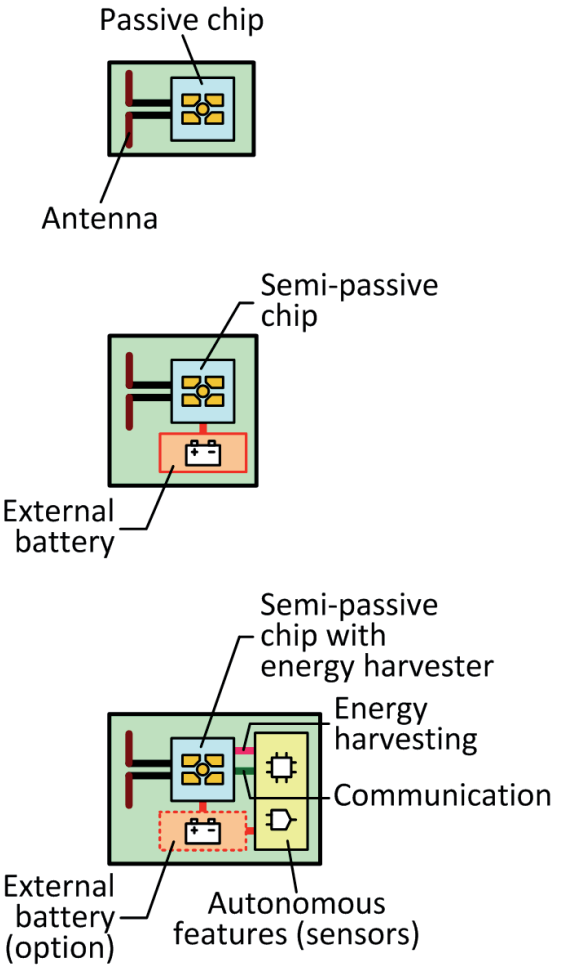

Fig. 1. Block diagrams of passive and semi-passive UHF transponders.

Semi-passive RFID transponders have built-in an extra supply source (e.g. lithium battery) which can be exchangeable or not. Generally it is used for enlarging the IZ, what is a very desirable feature for most implementation of RFID systems. Moreover, in some chips, the additional energy is used for powering blocks of supplementary autonomous functions, such as measuring physical quantities (humidity [14], [15], temperature [15-17], light intensity [15], [16], pressure [18], acceleration [19], gas [17], [20], etc.), writing gathered data into a built-in memory, etc. In the contemporary electronic market, the first new untypical RFID chips with the possibility of gathering the extra energy from the electromagnetic field emitted by RWD antennas have been announced. Usually, these very rare solutions can operate in a semi-passive mode (with battery) or in a passive mode (without battery). However, it should be kept in mind that the chips with the ability of recovering energy require a different antenna pattern than it is in the conventional passive transponders.

\section{Antenna Design}

\subsection{Impedance Matching}

The antenna impedance $Z_{T A}$ of the semi-passive UHF transponder with an energy harvester is constant at a given frequency, but the chip impedance $Z_{T C}$ varies while the transponder is working (Fig. 2).

The impedance matching of the chip and antenna is characterized by the power transfer coefficient $\tau$.

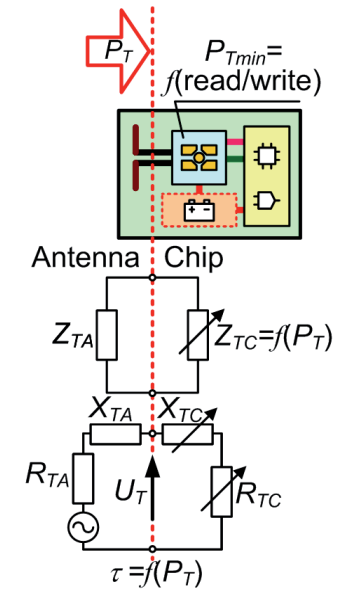

Fig. 2. Electrical equivalent diagram of antenna connected to semi-passive chip with energy harvester.

$$
\tau=\frac{4 \operatorname{Re}\left(Z_{T A}\right) \operatorname{Re}\left(Z_{T C}\right)}{\left(\operatorname{Re}\left(Z_{T A}+Z_{T C}\right)\right)^{2}+\left(\operatorname{Im}\left(Z_{T A}+Z_{T C}\right)\right)^{2}} .
$$

The value of impedance $Z_{T C}$ is dependent on a transponder localization and orientation relative to the RWD antenna plane. This dependency occurs because the impedance varies with the power $P_{T}$ which is transferred from the antenna to the chip [21]. Because of varying environmental and arrangement conditions the power level changes despite of using sophisticated rectifier and voltage regulator in the transponder RF front end [22]. These characteristics are crucial in the transponder antenna synthesis. The maximum range of RFID system is obtained for the full impedance matching of antenna and chip $\left(Z_{T A}=Z_{T C}\right.$, $\tau=1)$ at the minimal power $P_{T \min }$ (chip sensitivity) which is enough for activating internal circuits of the transponder. The $P_{T \min }$ is dependent on the type of chip and it describes supply conditions of the transponder.

The typical value of UHF semi-passive chip resistance $R_{T C}$ (Fig. 2) equals from a few to tens $\Omega$ [23], [24] at the chip sensitivity $P_{T m i n}$. The value of chip reactance $X_{T C}$ (typically a few of hundreds $\Omega$ ) depends mainly on an internal capacitance that accumulates energy which is necessary for supplying transponder. Impedance matching means that the chip resistance $R_{T C}$ is equal to the antenna resistance $R_{T A}$, and also the antenna reactance has inductive character - then the equation $Z_{T A}=Z_{T C}{ }^{*}$ is met.

\subsection{Antenna Construction for Semi-Passive Chip with Energy Harvester}

In the classical passive UHF chip, there are several ways to obtain highly inductive character of the antenna impedance [25].

The first group of methods consists in modification of microstrip antenna construction. It can be achieved by adjusting the coupling effect between antenna and transponder environment [26] or chip circuit, by utilizing T-matching (Fig. 3-a) [27] or parasitic induction loop (Fig. 3-b) and others. Sometimes, the use of these methods 


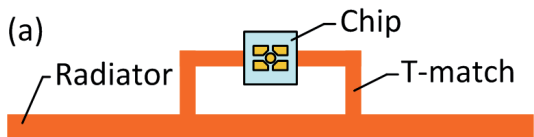

(b)

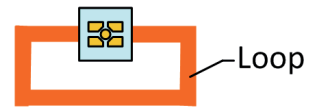

(c)

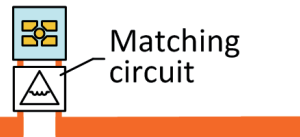

Fig. 3. Means of impedance matching in RFID transponder: a) T-match, b) parasitic loop, c) other antenna circuit.

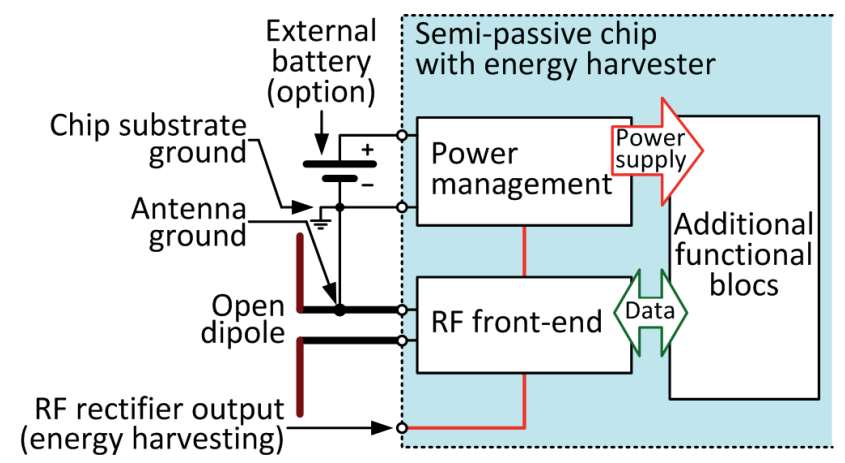

Fig. 4. Front-end in semi-passive chip with energy harvester.

is impossible for same solutions of chips because of their specific internal circuit design. Then it is necessary to use symmetrical/asymmetrical open-antenna arms (open dipole) which are adjusted by using a microstrip and/or SMD elements (Fig. 3-c) [28].

The considered type of chips has a RF rectifier output for gathering energy from the electromagnetic field of RFID system (Fig. 4). This output, together with the external battery (in the battery-assisted mode), is used for powering blocks of supplementary autonomous functions in a semi-passive transponder. The classical passive RFID antennas (utilizing e.g. T-matching or parasitic induction loop for matching impedance) negatively influence the harvester and lack of energy blocks the whole transmission between the transponder and RWD. It is due to the fact that antenna and chip substrate have a common ground. So, it is necessary to use the symmetrical/asymmetrical open dipole for semi-passive UHF RFID transponder with the energy harvester (Fig. 3-c). The mentioned method of impedance matching (using microstrip) have been utilized in test samples with the AMS chip.

\subsection{Design Assumptions}

The process of antenna design for the semi-passive UHF RFID transponder with the energy harvester is discussed on the basis of numerical calculations (model developed in the Mentor Graphics HyperLynx 3D EM) and practical implementations (in PCB technology). The investigation has been applied to the real RFID passive/semipassive chip (AMS SL900A in QFN16 package [23]).
Additionally, it is assumed in this work that the designed antenna should be resistant to the proximity of metal objects. This assumption stems directly from the fact that the construction of this kind of chips is the most advanced and the most expensive among the currently available on the market. This results in the need for labeling such transponders on objects of considerable value. During the process of freight forwarding guaranteeing that the valuable products will get to the proper destination by an agreed upon date, and in good condition it is necessary to control parameters of the surrounding environment by means of sensors e.g. embedded in the high performance transponders. On the other hand, it is impossible to use the typical low-cost transponders made in the form of pressure-sensitive labels in the disturbing environment (metal or any other object made of electrically conductive materials).

In the presented case, it is assumed that the designed antenna should have a directional radiation pattern, a small geometrical size and the power transfer coefficient $\tau=0.7: 1$ in the band: $865.6-867.6 \mathrm{MHz}$ and $902-928 \mathrm{MHz}$. The choice of the frequency band meets the requirement of proper operation in various parts of the world. It is especially important for long range RFID systems which work according to requirements of electronic product code in the UHF band (protocol ISO 18000-6c, RWD compatibility: a) European version of ETSI EN 302208 - 2 W ERP, frequency band 865.6-867.6 MHz, or b) American version of FCC Part 15.247 - $1 \mathrm{~W}$ of transmitter output power with maximal gain of $6 \mathrm{dBi}-4 \mathrm{~W}$ EIRP, frequency band $902 \mathrm{MHz}-928 \mathrm{MHz}$ ).

\subsection{Model}

On the basis of [28], the measured values of the SL900A chip impedance are presented in Tab. 1 for passive (without external battery) and semi-passive mode (with $1.5 \mathrm{~V}$ external battery). The values are obtained at the measured sensitivity $P_{T m i n}$.

\begin{tabular}{|c|c|c|}
\hline $\begin{array}{c}f_{0} \\
\mathrm{MHz}\end{array}$ & $\begin{array}{c}Z_{T C}, \Omega \\
\text { passive mode }\end{array}$ & $\begin{array}{c}Z_{T C}, \Omega \\
\text { semi-passive mode }\end{array}$ \\
\hline 866 & $14.9-\mathrm{j} 342 \mid P_{T \min }=-13.1 \mathrm{dBm}$ & $14.7-\mathrm{j} 338 \mid P_{T_{\min }}=-14.8 \mathrm{dBm}$ \\
\hline 915 & $15.3-\mathrm{j} 313 \mid P_{T \min }=-13.4 \mathrm{dBm}$ & $14.9-\mathrm{j} 309 \mid P_{T \min }=-14.9 \mathrm{dBm}$ \\
\hline
\end{tabular}

Tab. 1. Results of impedance measurements for SL900A chip.

It should be noted that the impedance value at the power $P_{T \min }$ is independent of the supplementary battery source. This fact has essential practical meaning because it allows designers to construct only one type of transponder antenna for the both operating modes (passive and semipassive). The knowledge of chip impedance exact value at given frequency is crucial for determining matching efficiency i.e. coefficient $\tau$. The chip impedance is assigned at sensitivity $P_{\text {Tmin }}=-15 \mathrm{dBm}$ (Fig. 5). This value represents a compromise looking at the frequency band 860-960 MHz and passive or semi-passive mode [28]. The proposed design of transponder antenna is presented in Fig. 6. The numerical model of the pattern has been prepared in HL3DEM. 


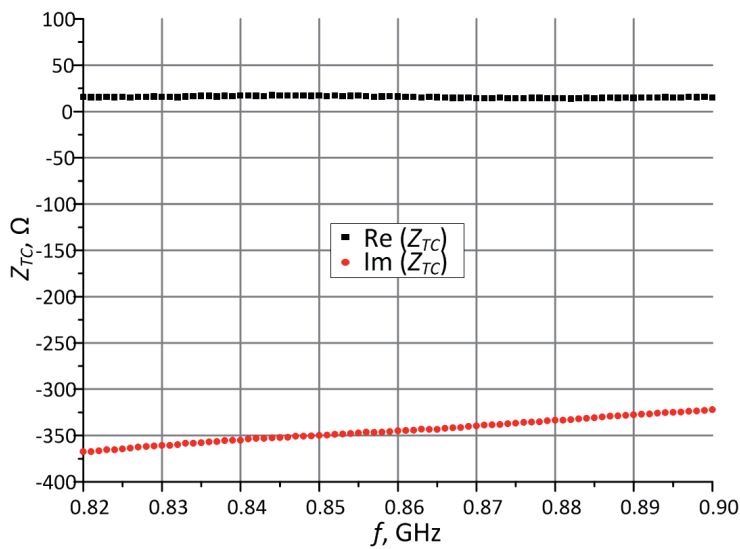

Fig. 5. Measured characteristic of SL900A chip impedance.

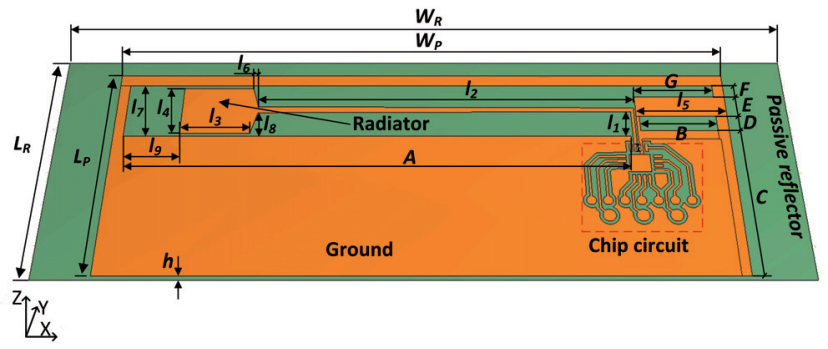

Fig. 6. Design of transponder antenna; HL3DEM model.

The radiator with arms of open antenna, the ground and the chip electrical circuit are in the upper layer while the structure of the lower layer consists of a passive reflector. The radiating element and the chip outputs are surrounded by a ground layer. It reduces the antenna sensitivity to the influence of environmental conditions on the sides and front of the transponder. The location of the passive reflector under the radiator ensures proper parameters of antenna regardless of the labeled object type (its size and material).

Dimensions of each antenna edge depend primarily on the resonance frequency $f_{0}$ and the relative permittivity $\varepsilon_{r}$ of the dielectric layer that is used in the copper clad laminate. Therefore the low-loss double-sided laminate ISOLA IS-680-300 (thickness of dielectric layer $h=1.547 \mathrm{~mm}$, thickness of copper layer $18 \mu \mathrm{m}, \varepsilon_{r}=3$, $\operatorname{tg} \delta=0.003$ at $f_{0}=2 \mathrm{GHz}$ ) are used to prepare samples according to the elaborated antenna design. Due to the lack of reliable information about the parameters of the dielectric layer for the presumed resonance frequency $(866 \mathrm{MHz}$ and $915 \mathrm{MHz}$ ) additional tests have to be carried out. The proper value of $\varepsilon_{r}=3.08$ necessary in the model calculation is determined on the basis of ring resonators.

The calculation process in HL3DEM simulator is time consuming but it can be executed in successive stages. It allows designers to introduce construction modifications into the calculated model especially with regards to complex parameters (frequency band, power transfer coefficient, radiation pattern and its parameters, etc). It is possible by changing variables manually or numerically in the optimization process. The automatic parameter modifications are provided by adaptive EM procedure which com- bines the advantages of local and global optimizer and other advanced technology of simulation software environment to achieve optimized performance with the fewest EM simulations.

A lot of different cases of objects proximity are taken into consideration during simulations. Special attention is paid to objects made of metal which by their nature the most often disturb the electromagnetic field in the practical use of RFID systems. On the basis of obtained results, it can be found that the use of passive reflector and ground layer around the radiator significantly improves parameter stability (power transfer coefficient, radiation pattern and its parameters) of the designed antenna to the acceptable level in real implementations.

Therefore final dimensions of the antenna: $W_{R}=116 \mathrm{~mm}, L_{R}=39 \mathrm{~mm}, W_{P}=96.6 \mathrm{~mm}, L_{P}=35 \mathrm{~mm}$, $A=79 \mathrm{~mm}, \quad B=12 \mathrm{~mm}, \quad C=24.9 \mathrm{~mm}, \quad D=2.7 \mathrm{~mm}$, $E=3.6 \mathrm{~mm}, \quad F=2.2 \mathrm{~mm}, \quad G=12.7 \mathrm{~mm}, \quad l_{1}=4.5 \mathrm{~mm}$, $l_{2}=59 \mathrm{~mm}, \quad l_{3}=11 \mathrm{~mm}, \quad l_{4}=8.3 \mathrm{~mm}, \quad l_{5}=14.4 \mathrm{~mm}$, $l_{6}=0.98 \mathrm{~mm}, l_{7}=9.4 \mathrm{~mm}, l_{8}=4.4 \mathrm{~mm}, l_{9}=8.7 \mathrm{~mm}$ represents a compromise looking at the design assumptions (Sec. 3.3). The results confirm the validity of assumptions made for the proposed antenna of semi-passive UHF RFID transponder with the energy harvester.

The test samples of elaborated antenna project are made on the basis of the elaborated numerical model (Fig. 7). The proposed solution provides basis for further development work (Grant No. PBS1/A3/3/2012) conducted in the scope of RFID technique. The idea of multiband (UHF, HF) development board dedicated to battery-less autonomous semi-passive RFID transponders is approached in the further stage (Fig. 8).

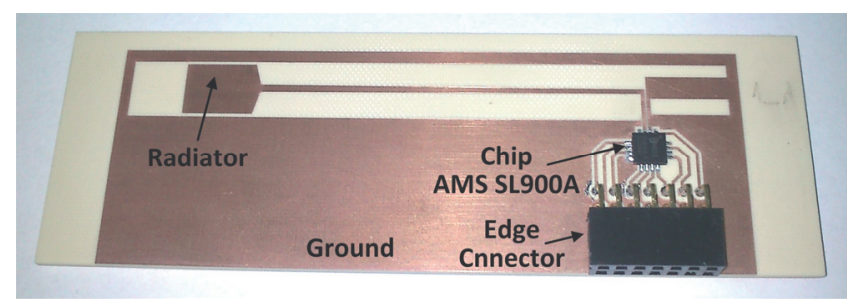

Fig. 7. Antenna sample - practical implementation.

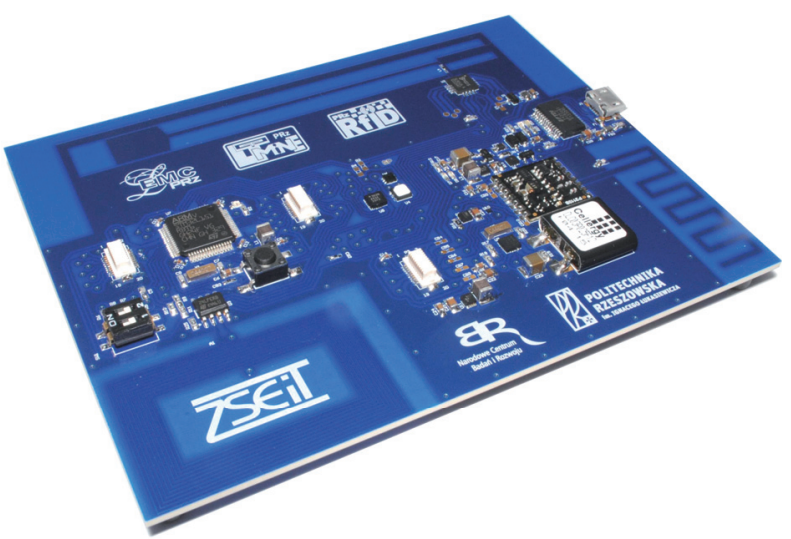

Fig. 8. Development board of the autonomous semi-passive RFID transponder. 


\subsection{Results}

The calculations and measurements are carried out for the impedance matching at the frequency $f_{0}=866 \mathrm{MHz}$ and $915 \mathrm{MHz}$. The results obtained for antenna impedance are summarized in Fig. 9. The calculations and measurements carried out for the power transfer coefficient are presented in Fig. 10. $\tau$ is calculated on the basis of (1) and the chip impedance $Z_{T C}$ which is measured at sensitivity $P_{T \min }=$ $-15 \mathrm{dBm}$ (Fig. 5). The impedance parameters of the chip and antenna are measured by using VNA Agilent PNA-X N5242A Vector Network Analyzer and PacketMicro TDP10210 differential probe. The probe is calibrated by using PacketMicro TCS50 RF Calibration Substrate [29].
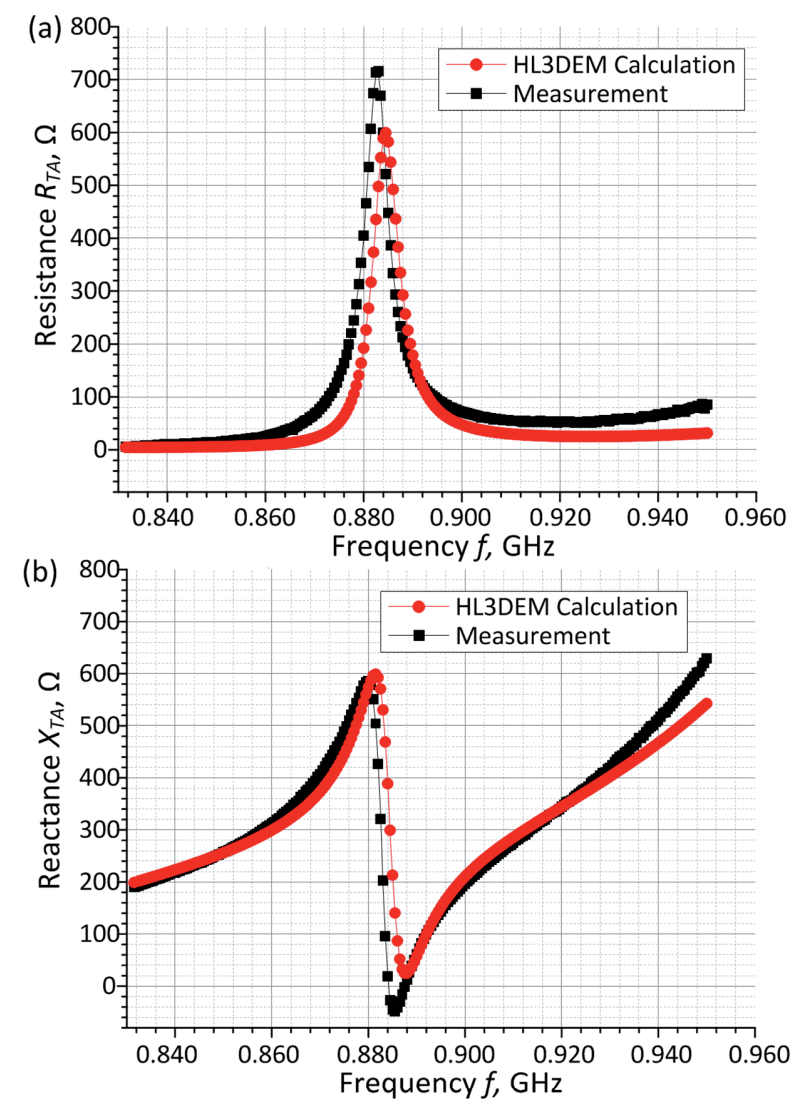

Fig. 9. Antenna impedance: a) resistance, b) reactance.

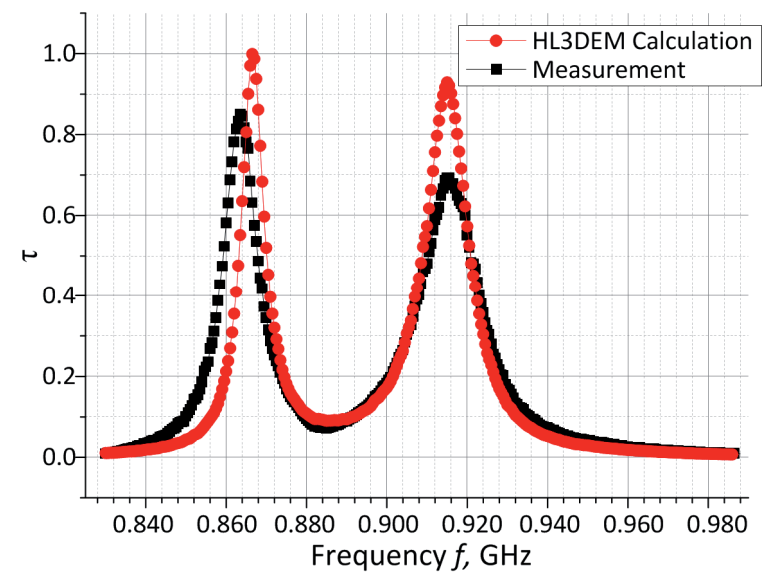

Fig. 10. Power transfer coefficient.

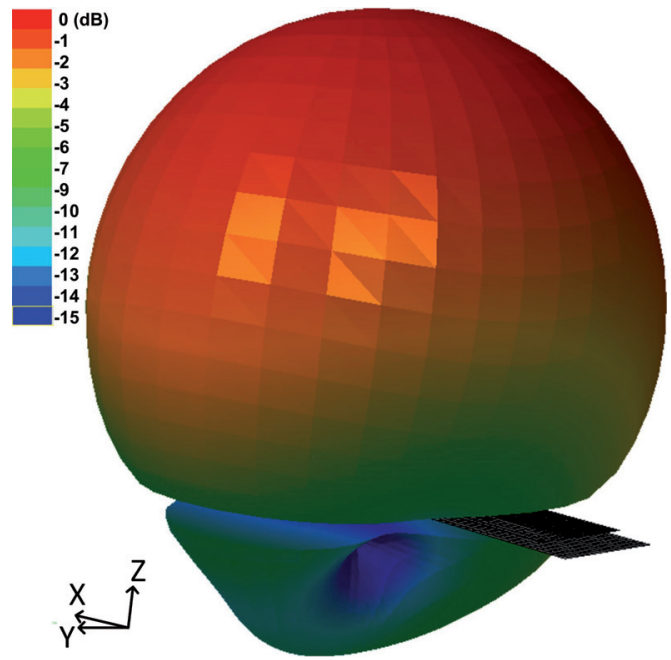

Fig. 11. 3D view of calculated radiation pattern for $866 \mathrm{MHz}$.
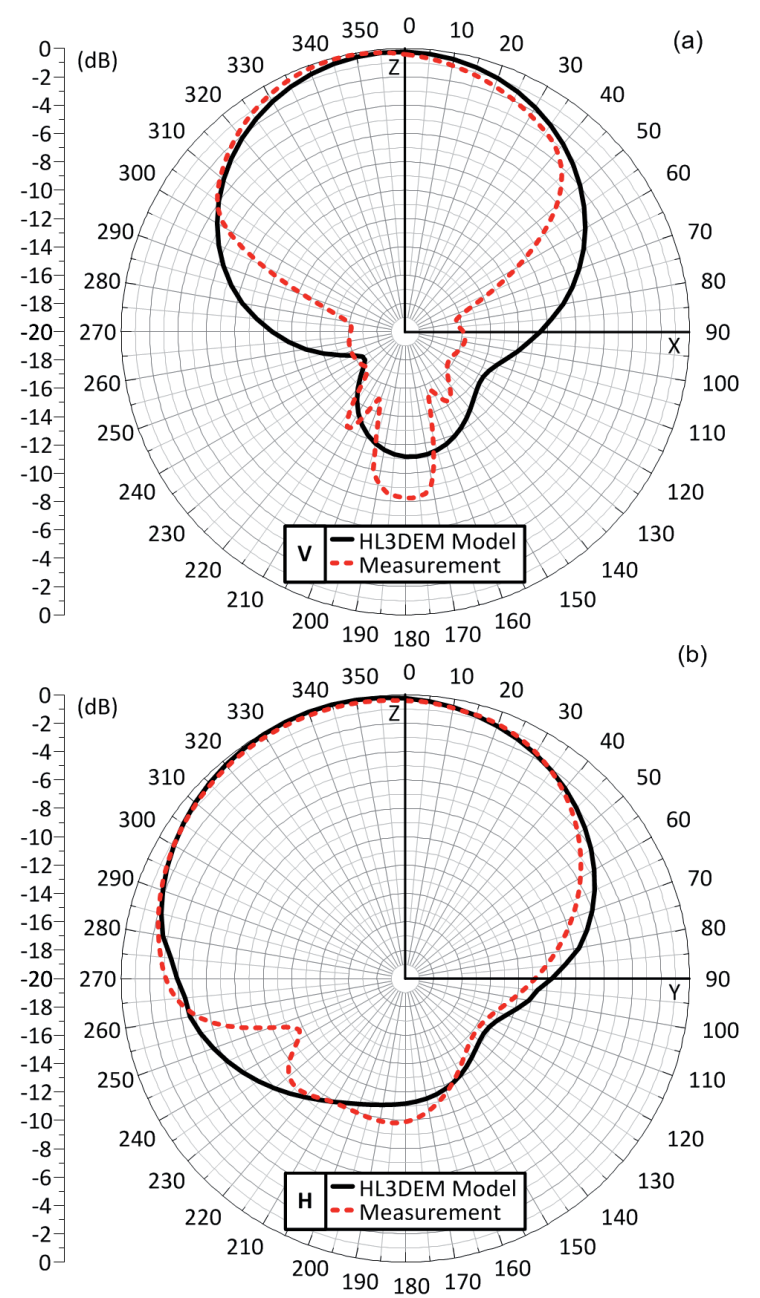

Fig. 12. Charts of antenna radiation pattern for $866 \mathrm{MHz}$ : a) V plane, b) H plane.

In the presented design project, the measured maximal values of power transfer coefficient are less than that obtained with numerical calculations of about 0.2 (for $\tau=0.996$ at $f_{0}=866 \mathrm{MHz}, \tau=0.928$ at $\left.f_{0}=915 \mathrm{MHz}\right)$. From a practical point of view, the convergence of the results is satisfied. 
The $3 \mathrm{D}$ calculated radiation pattern of the designed antenna is presented in Fig. 11. The comparison of measured and calculated characteristic diagrams are listed in a V-vertical (X-Z) and H-horizontal (Y-Z) plane (Fig. 12).

The measurements were performed in a TDK anechoic chamber with using dedicated equipment. The main apparatus are especially adapted to the specified investigations. It was necessary to design SysAntPat software tool in LabView dedicated to azimuth positioner Dream Catcher ME1300, an also to adjust the IDS R903 UHF RFID read/write device that is used unconventionally as an indicator of the received signal strength level.

On the basis of result analysis it should be noticed that the vast majority of the energy radiated by the antenna is concentrated in the main beam. The calculated radiated efficiency equals $44 \%$ at $f_{0}=866 \mathrm{MHz}$ and $48 \%$ at $f_{0}=915 \mathrm{MHz}$ (the maximum value of the gain is, respectively, $0.84 \mathrm{dBi}$ and $1.23 \mathrm{dBi}$ ). This means that the passive reflector fulfills its role.

Additionally, it can be noticed that the convergence of characteristics is high for the main lobe and satisfactory for the rest of directions. The minor discrepancies are caused by using an infinite plane for the dielectric layer in the prepared HL3DEM numerical model.

\section{Conclusion}

From the user point of view, the most important factor of the RFID system is the efficiency of automated identification process. This means that electronically tagged objects placed in any point of the operation space have to be recognized correctly with $100 \%$ certainty in the best case. Only properly performed design of antenna system gives possibility to meet this requirement. The correct estimation of the IZ is only possible when the knowledge base of the component parameters is complete. It should be emphasized that there is not a universal transponder available on the market for each kind of applications - the standard solutions are adequate for limited number of automated identification processes. For other cases, the transponder antenna should be always designed according to specific requirements of the given implementation. But the progress in electronics gives new opportunity. The innovative RFID chip designs with additional functions implemented inside (e.g. extra harvester, sensors) broaden operating range of the whole RFID technique. Unfortunately, the antenna for such a chip has to be designed differently than it is in the case of standard passive transponders. Considering these aspects, the authors focused on the synthesis of useful microstrip antenna design for a semi-passive UHF RFID transponder with the energy harvester that operates in accordance with the requirements of the Electronic Product Code (EPC Class 1 Gen 2, ISO/IEC 18000-6c). The particular attention was paid to preparing the model of the untypical radiating structure because the considered type of chips has a RF rectifier output for gathering energy from the electromagnetic field of RFID system (antenna and chip have a common ground). This process is crucial for the efficient implementation of the contemporary research and development projects (autonomous semi-passive transponder). So, it is necessary to use only the symmetrical/ asymmetrical open dipole for semi-passive UHF transponder where harvested energy from the electromagnetic field of RFID system will be used for powering blocks of supplementary autonomous functions.

\section{Acknowledgments}

This work was supported in part by the Polish National Centre for Research and Development (NCBR) under Grant No. PBS1/A3/3/2012. The work was developed by using equipment purchased in the Operational Program Development of Eastern Poland 2007-2013 of the Priority Axis I Modern Economics of Activity I.3 Supporting Innovation under Grant No. POPW.01.03.00-18-012/09-00 and the Program of Development of Podkarpacie Province of the European Regional Development Fund under Grant No. UDA-RPPK.01.03.00-18-003/10-00.

\section{References}

[1] FINKENZELLER, K. RFID Handbook. $3^{\text {rd }}$ ed., Wiley, 2010. ISBN: 9780470695067

[2] BROWN, D. RFID Implementation. McGraw-Hill, 2007. ISBN: 9780072263244

[3] CHEN, J. C., CHENG, C.-H., HUANG, P. B., WANG, K.-J., HUANG, C.-J., TING, T.-C. Warehouse management with lean and RFID application: a case study. International Journal of Advanced Manufacturing Technology, 2013, vol. 69, no. 1-4, p. 531-542. DOI: $10.1007 / \mathrm{s} 00170-013-5016-8$

[4] JONES, E., CHUNG, C. RFID in Logistics - A Practical Introduction. CRC, 2008. ISBN: 9780849385261

[5] KOCHENDÖRFER, P. Entering a secure future. Global Identification, Oct. 2012, p. 24-26.

[6] WHEELER, M. Automating processes with RFID. Global Identification, Feb. 2013, p. 10-13.

[7] JANKOWSKI-MIHUŁOWICZ, P., KALITA, W., PAWŁOWICZ, B. Problem of dynamic change of tags location in anticollision RFID systems. Microelectronics Reliability, 2008, vol. 48, no. 6, p. 911-918. DOI: 10.1016/j.microrel.2008.03.006

[8] SHAO, S., BURKhOlder, R. J., VOLAKIS, J. L. Design approach for robust UHF RFID tag antennas mounted on a plurality of dielectric surfaces. IEEE Antennas and Propagation Magazine, 2014, vol. 56, no. 5, p. 158-166. DOI: 10.1109/MAP.2014.6971931

[9] GOUdOS, S. K., SIAKAVARA, K., SAHALOS, J. N. Novel spiral antenna design using artificial bee colony optimization for UHF RFID applications. IEEE Antennas and Wireless Propagation Letters, 2014, vol. 13, p. 528-531. DOI: 10.1109/LAWP.2014.2311653

[10] CHOO, J., RYOO, J. UHF RFID tag applicable to various objects. IEEE Transactions on Antennas and Propagation, 2014, vol. 62, no. 2, p. 922-925. DOI: 10.1109/TAP.2013.2290051 
[11] SUN, X.-B., XIE, J., CAO, M.-Y. RFID tag antenna design based on an improved coupling source shape. IEEE Antennas and Wireless Propagation Letters, 2013, vol. 12, p. 532-534. DOI: 10.1109/LAWP.2013.2255856

[12] ZAMORA, G., ZUFFANELli, S., PAREDES, F., MARIN, F., BONACHE, J. Design and synthesis methodology for UHF-RFID tags based on the T-match network. IEEE Transactions on Microwave Theory and Techniques, 2013, vol. 61, no. 12, p. 4090-4098. DOI: 10.1109/TMTT.2013.2287856

[13] GS1 EPCglobal. EPC Radio-Frequency Identity Protocols Generation-2 UHF RFID; Specification for RFID Air Interface Protocol for Communications at $860 \mathrm{MHz}-960 \mathrm{MHz}$. Ver. 2.0.0, Nov-2013. [Online] Available at: http://www.gs1.org/epcglobal

[14] PHAN, N. D., CHANG, I. J., LEE, J. W. A 2-Kb one-time programmable memory for UHF passive RFID tag IC in a standard $0.18 \mu \mathrm{m}$ CMOS process. IEEE Transactions on Circuits and Systems I, 2012, vol. 60, no. 7, p. 1810-1822. DOI: 10.1109/TCSI.2012.2230500

[15] SLOSARČÍK, S., VEHEC, I., KALITA, W., BAUER, R., SABAT, W. 3D shaped module with integrated pressure sensor ATP Journal plus, 2007, vol. 1, p. 228-230. ISSN: 1336-5010 (in Slovak).

[16] LEE, C. W., LEE, S. J., KIM, M., KYUNG, Y., EOM, K. Capacitive humidity sensor tag smart refrigerator system using the capacitive to voltage converter (CVC). International Journal of Science and Advanced Technology, 2011, vol. 36, p. 15-26. ISSN: 2221-8386

[17] ABAD, E., MAZZOLAI, B., JUARROS, A., GÓMEZ, D., MONDINI, A., SAYHAN, I., KRENKOW, A., BECKER, T. Fabrication process for a flexible tag microlab. Proceedings of SPIE, vol. 6589, Smart Sensors, Actuators, 2007, 65890O. DOI: $10.1117 / 12.723737$

[18] OPREA, A., BÂRSAN, N., WEIMAR, U., BAUERSFELD, M. L., EBLING, D., WÖLLENSTEIN, J. Capacitive humidity sensors on flexible RFID labels. Sensors and Actuators B, 2008, vol. 132, no. 2, p. 404-410. DOI: 10.1016/j.snb.2007.10.010

[19] VOLK, T., JANSEN, D., SPELETZ, H., FLEINER, B., BAU, D., KREKER, A., RISKE, A. Active RFID sensor with integrated file system for logistic applications. In Proceedings of 2010 European Workshop on Smart Objects: Systems, Technologies and Applications. Ciudad (Spain), 2010, p. 1-7. ISBN: 9783800732821

[20] KIM, S., CHO, J. H., KIM, H. S., KIM, H., KANG, H. B., HONG, S. K. An EPC Gen 2 compatible passive/semi-active UHF RFID transponder with embedded FeRAM and temperature sensor. In Proc. of IEEE Asian Solid-State Circuits Conference. Jeju (South Korea), 2007. p. 135-138. DOI: 10.1109/ASSCC.2007.4425750

[21] DE VITA, G., IANNACCONE, G. Design criteria for the RF section of UHF and microwave passive RFID transponders. IEEE Transactions on Microwave Theory and Techniques, 2005, vol. 53, no. 9, p. 2978-2990. DOI: 10.1109/TMTT.2005.854229

[22] WEI, P., CHE, W., BI, Z., WEI, C., NA, Y., QIANG, L., HAO, M. High-efficiency differential RF front-end for a Gen2 RFID tag. IEEE Transactions on Circuits and Systems II, 2011, vol. 58, no. 4, p. 189-194. DOI: 10.1109/TCSII.2011.2124530

[23] AMS. SL900A EPC Class 3 Sensory Tag Chip - For Automatic Data Logging. AMS Datasheet, 2014-May-06, v1-01.

[24] RAMTRON. WM72016 - 16Kbit Secure F-RAM Memory with Gen-2 RFID Access \& Serial Port Direct Memory Access. Product Specification, Rev. 3.0, Sep. 2012.

[25] MARROCCO, G. The art of UHF RFID antenna design: impedance-matching and size-reduction techniques. IEEE Antennas and Propagation Magazine, 2008 vol. 50, no. 1, p. 66-79. DOI: 10.1109/MAP.2008.4494504

[26] KIM, D, YEO, J. Dual-band long-range passive RFID tag antenna using an AMC ground plane. IEEE Transactions on Antennas and
Propagation, 2012, vol. 60, no. 6, p. 2620-2626. DOI: 10.1109/TAP.2012.2194638

[27] MOHAMMED, N. A., DEMAREST, K., DEAVOURS, D. D. Analysis and synthesis of UHF RFID antennas using the embedded T-match. In Proceedings of the IEEE International Conference on RFID. Orlando, (FL, USA), 2010, p. 230-236. DOI: $10.1109 /$ RFID.2010.5467276

[28] JANKOWSKI-MIHUŁOWICZ, P., WEGLARSKI, M. Determination of passive and semi-passive chip parameters required for synthesis of interrogation zone in UHF RFID systems. Elektronika ir Elektrotechnika (Electronics and Electrical Engineering), 2014, vol. 20 , no. 9 , p. 65-73. DOI: 10.5755/j01.eee.20.9.5007

[29] JANKOWSKI-MIHUŁOWICZ, P., PITERA, G., WEGLARSKI, M. The impedance measurement problem in antennas for RFID technique. Metrology and Measurement Systems, 2014, vol. XXI, no 3, p. 509-520. DOI: 10.2478/mms-2014-0043

\section{About the Authors ...}

Piotr JANKOWSKI-MIHULOWICZ was born in 1974. He received the MSc. degree in the specialization of Electronic Devices from the Faculty of Electrical Engineering, Rzeszów University of Technology (Poland), in 1999, and the Ph.D. degree in the field of Telecommunications at AGH University of Science and Technology, in 2007. Since 2007, he has been an Assistant Professor with the Dept. of Electronic and Communications Systems (ZSEiT), Faculty of Electrical and Computer Engineering, Rzeszów University of Technology. He is the author of numerous publications and studies for industry. His scientific research is focusing on antennas, RFID systems and their practical uses. He co-operates with companies in the country and abroad in order to solve problems from the range of RFID technology. Dr. Jankowski-Mihułowicz is a Member of IEEE, IMAPS and SEP.

Damian KAWALEC received his BSc. and MSc. diploma in Electronics and Telecommunications from Rzeszów University of Technology in 2009 and 2011, respectively. Since 2013 he participates in research project "Synthesis of autonomous semi-passive transponder dedicated to operation in anti-collision dynamic RFID systems". His research interests include antenna designing, EM wave propagation and modeling of RFID systems. Currently he is a $\mathrm{PhD}$ student in the Dept. of Electronic and Communications Systems, Rzeszów University of Technology.

Mariusz WEGLARSKI was born in 1971. In 1996 he graduated (MSc.) in the specialization of Electronic Devices from the Faculty of Electrical Engineering, Rzeszów University of Technology, Poland. He defended his PhD thesis in the field of Electrical Engineering at the Faculty of Electrical and Computer Engineering, Rzeszów University of Technology, in 2005. Since 1996, he has been an Assistant Professor in the Dept. of Electronic and Communications Systems, Rzeszów University of Technology. He is the author of more than 50 publications. His scientific research is focusing on hybrid microelectronic and microsystem technology, analysis of temperature fields, RFID systems technology and their applications. Dr. Węglarski is a Member of IEEE, IMAPS and SEP. 